\title{
La visualisation de données en éducation
}

\author{
Federica Minichiello
}

\section{(2) OpenEdition}

Journals

Édition électronique

URL : http://journals.openedition.org/ries/3949

DOI : $10.4000 /$ ries.3949

ISSN : 2261-4265

\section{Éditeur}

Centre international d'études pédagogiques

\section{Édition imprimée}

Date de publication : 1 septembre 2014

Pagination : 10-14

ISBN : 978-2-85420-604-3

ISSN : 1254-4590

\section{Référence électronique}

Federica Minichiello, "La visualisation de données en éducation », Revue internationale d'éducation de Sèvres [En ligne], 66 | septembre 2014, mis en ligne le 01 septembre 2014, consulté le 22 juin 2020. URL : http://journals.openedition.org/ries/3949 ; DOI : https://doi.org/10.4000/ries.3949

Ce document a été généré automatiquement le 22 juin 2020.

(c) Tous droits réservés 


\title{
La visualisation de données en éducation
}

\author{
Federica Minichiello
}

1 Une "visualisation de données" est une combinaison d'éléments graphiques, accompagnée d'analyses statistiques, qui donne du relief à des éléments d'information difficilement saisissables dans un texte écrit. La visualisation fait partie intégrante d'un processus d'analyse des données et offre à l'utilisateur la possibilité d'explorer des chiffres, en lui proposant des clés pour une lecture critique et, parfois, l'opportunité de découvrir des éléments inattendus.

2 La représentation graphique n'est pas une invention récente mais on assiste à un intérêt croissant à son égard, dans les publications institutionnelles et dans les médias, en raison d'une meilleure accessibilité aux données (parfois très volumineuses) et aux technologies graphiques sous-jacentes.

Dans la première partie de cet article, nous présentons des ressources qui décrivent la visualisation de données dans sa double nature graphique et statistique. Nous proposons ensuite quelques exemples de réalisations dans le domaine éducatif et nous terminons par une sélection d'outils pour s'entraîner à « visualiser » les données.

4 Pour entrer dans le cœur du sujet, nous avons choisi d'accompagner ce texte d'introduction par deux ressources vidéo très intéressantes graphiquement. La première est "Story of the world", le défi du statisticien Hans Rosling de raconter l'histoire du monde en quatre minutes: « 200 pays, sur 200 années, en utilisant 120000 chiffres » (disponible sur le site de la $\mathrm{BBC}$ ); la deuxième est le documentaire d'Inge Druckrey «Teaching to see ", produit par Edward Tufte (surnommé le « Léonard de Vinci des données »), qui rappelle que la force de toutes ces réalisations et technologies réside dans la capacité humaine à voir et à lire une image ${ }^{1}$.

Sitographie arrêtée le 30 juin 2014. 


\section{La visualisation des données}

\section{"Visualisation de données - les leçons de l'Histoire »}

5 Cette présentation, proposée lors de la semaine européenne de l'Open Data en $2012^{2}$, offre un regard historique sur la visualisation de données et situe cette tendance actuelle dans le cadre d'une discipline rigoureuse, ponctuée par les travaux en sémiologie graphique du cartographe Jacques Bertin ${ }^{3}$ et les analyses de William S. Cleveland et Robert McGill ${ }^{4}$ sur l'efficacité des différentes formes graphiques. Il est également rappelé que, si les évolutions d'aujourd'hui vont vers une croissante créativité, cela se fait parfois au détriment d'une analyse rigoureuse des données. Présentation disponible sur le site http://www.opendataweek.org/2012 [en français]

\section{Veille « Journalisme graphique »}

6 Karen Bastien, data-journaliste française (http://www.wedodata.fr/) met à disposition une veille sur le journalisme graphique et la visualisation de données sur la plateforme de partage scoop.it. Elle recense des centaines de réalisations graphiques multidisciplinaires, produites par des institutions, des journalistes ou des particuliers, comme par exemple "Lexical distance among-languages of Europe", une illustration cartographique de la distance lexicale entre les principales langues européennes, sur la base du degré de divergence entre les différents vocabulaires ${ }^{5}$.

http://www.scoop.it/t/journalisme-graphique [en français]

\section{"The Functional Art »}

7 "The Functional Art» est un ouvrage de référence sur la visualisation de données d'Alberto Cairo, professeur en graphisme de l'information à l'Université de Miami et coordinateur pédagogique des premiers cours en ligne ouverts à tous («MOOCs») sur le journalisme de données. Le site homonyme répertorie de nombreuses ressources qui traitent de la visualisation et définissent ce qui caractérise une représentation pertinente: la beauté graphique, l'utilité de l'information et la capacité d'éclairer le lecteur.

http://www.thefunctionalart.com/ [en anglais]

\section{The Office For Creative Research}

Le "Office For Creative Research" est un groupe de recherche multidisciplinaire qui explore les possibilités d'utilisation des données par le biais de pratiques innovantes qui se situent "à l'intersection de la science, de l'art et du design». L'un de ses fondateurs est Jer Thorp, qui a travaillé dans l'équipe de recherche et développement du New York Times entre 2010 et 2012. Le site du groupe de recherche et son blog personnel présentent une sélection de réalisations de l'artiste, dont la philosophie est d'« humaniser » les données et de les utiliser comme point de départ pour une création artistique.

http://o-c-r.org/; http://blog.blprnt.com/ [en anglais] 


\section{La visualisation appliquée aux données en éducation}

9 Dans cette section, nous présentons plusieurs typologies de visualisation dans le domaine éducatif. L'ordre logique de leur présentation est la diversité de la finalité visée : nous partons de la simple description ou information de contexte, très courante dans les publications institutionnelles, pour aller vers des réalisations graphiques plus innovantes, qui permettent de découvrir une nouvelle information ou de divulguer aux lecteurs des informations complexes, en leur proposant une réelle appropriation des données.

\section{Le rapport mondial de suivi sur l'Éducation pour tous (UNESCO)}

10 Le rapport mondial de suivi sur l'Éducation pour tous est une publication de référence qui vise à « informer, influencer et à soutenir l'engagement mondial en faveur de l'éducation pour tous ». Ce travail est aussi à l'origine de nombreuses infographies, comme par exemple les représentations du World Inequality Database on Education (WIDE) sur les niveaux d'inégalité en éducation. Un autre exemple est « L'Éducation transforme nos existences ", une série de neuf infographies publiées dans le rapport 2013, qui illustrent le rôle de l'éducation en tant que «catalyseur pour réaliser les objectifs généraux du développement » et ses impacts sur la croissance économique, la mortalité maternelle, le développement de la tolérance, etc.

http://www.education-inequalities.org/ [en anglais]

http://www.education-transforms.org/ [en anglais, français, espagnol]

\section{La Banque mondiale : Edstats}

11 Le site «EdStats » de la Banque mondiale s'appuie sur une base de données centralisée des statistiques de l'éducation, provenant de plusieurs sources internationales dont l'Institut de statistique de l'UNESCO. Les visualisations proposées visent une finalité descriptive mais permettent aux utilisateurs une lecture très personnalisée. Il est possible d'étudier la performance d'un pays ou d'une région sur une variété d'indicateurs clés (les dépenses d'éducation, le taux de scolarisation, le taux d'alphabétisation, le ratio élève-enseignants, etc.) et d'explorer les données à travers des cartes géographiques, des graphiques dynamiques et des tableaux.

http://datatopics.worldbank.org/education/ [en anglais]

\section{OCDE : « Education at Glance » et PISA}

12 L'OCDE compte à son actif une production graphique très importante mais son exemple est particulièrement intéressant car l'institution a lancé, en septembre 2012, un concours grand public pour représenter le retour sur investissement dans l'éducation, sur la base des données issues du rapport «Education at Glance ». L'infographie lauréate ${ }^{6}$ a réussi à proposer un regard nouveau et à transcrire l'interaction complexe entre coûts et rendements, dans un support graphique facile, qui se prête à la comparaison entre pays et secteurs (public et privé).

En juin 2014, un nouveau concours a été lancé, à partir des données de l'enquête PISA, sur deux thèmes: les inégalités dans la réussite scolaire et la corrélation entre les 
conditions de scolarisation et la performance. Le site « PISA contest » répertorie toutes les réalisations en lice, qui illustrent, par exemple, la relation entre les résultats en mathématiques et la pénurie d'enseignants, l'impact de l'âge de scolarisation sur les résultats scolaires, etc.

«OECD Announces Winner of Global Data Visualisation Competition» sur http:// www.oecd.org [en anglais] ; http://beta.icm.edu.pl/PISAcontest/ [en anglais]

\section{La visualisation comme outil de pilotage}

L'Institute for Public School Initiatives de l'Université du Texas a organisé, en mai 2014, le premier colloque sur la visualisation des données en éducation, afin d'interroger des experts en graphisme et des éducateurs sur ses perspectives d'utilisation en tant qu'instrument de pilotage du système éducatif. Un exemple est le travail de représentation du bien être des élèves de l'Education Policy Center de l'Université du Nouveau Mexique: grâce à une fine transposition des indicateurs nationaux sur un territoire ciblé et au croisement avec d'autres indicateurs territoriaux, le centre a réussi à produire une série de cartographies recentrées sur le contexte local, ses enjeux et ses facteurs de réussite, comme par exemple le bilinguisme ${ }^{7}$.

http://www.edvis.org/ [en anglais] ; http://cepr.unm.edu/ [en anglais]

\section{Deux exemples dans le data-journalisme ${ }^{8}$}

\section{« In climbing income ladder location matters » (New York Times)}

15 Ce premier exemple issu du data-journalisme s'appuie sur un projet de recherche financé par la National Science Foundation: "Is America the "Land of Opportunity"?" (http://www.equality-of-opportunity.org/), qui analyse la variation de la mobilité sociale et sa relation avec plusieurs facteurs locaux dont la distribution des revenus et l'offre éducative. Les résultats de l'étude suggèrent que la mobilité ascendante tend à être plus élevée dans les régions métropolitaines, où les familles à faible revenu sont davantage dispersées, que dans les quartiers à revenu mixte. L'équipe du New York Times a réalisé un article sur le sujet, accompagné de visualisations qui facilitent la comparaison et permettent de lire les résultats de façon presque immédiate.

http://www.nytimes.com/ [en anglais] [consulté le 22 juillet 2013]

\section{"Education cuts interactive: what are the consequences? " (The Guardian)}

Le quotidien anglais The Guardian publie dans la rubrique "Show and Tell » une sélection de la meilleure actualité en termes de visualisation de données. En guise d'exemple, on peut citer "Education cuts interactive: what are the consequences", une visualisation de l' Oxford Home schooling en réponse à l'annonce de restrictions budgétaires publiques en éducation. L'infographie est une construction interactive en lego: chaque brique représente une typologie de dépense ; un affichage en surbrillance permet de montrer la part de réduction préconisée par la réforme. Les lecteurs peuvent ainsi explorer les données et construire leur propre opinion sur l'actualité du moment. http://www.theguardian.com [en anglais] [consulté le 30 octobre 2013] 


\section{Les perspectives}

La représentation graphique peut se révéler un puissant point de départ pour amorcer un débat. Si la visualisation dépend entièrement d'une analyse pertinente des données, la facilité de prise en main des outils graphiques explique la popularité de ces réalisations visuelles. Nous avons choisi de terminer cet article par une petite «boite à outils ", qui permettra de découvrir la visualisation dans ses aspects le plus pratiques.

\section{Dataviz catalogue}

Ce catalogue, né d'un projet individuel de gestion de connaissances dans la visualisation des données, s'est transformé au fur et à mesure en un outil de recensement de plusieurs typologies de représentation, qui décrit leur logique de construction et les répertorie en fonction de la finalité visée : faire une comparaison, représenter une hiérarchie, une relation, etc.

http://www.datavizcatalogue.com/ [en français]

\section{LibGuide « Data visualization », Duke University}

19 Le centre de ressources documentaires de la Duke University, aux États-Unis, propose une liste hiérarchisée de typologies de visualisations, organisée sur la base d'une taxonomie dédiée et présente pour chaque forme graphique des exemples et les outils techniques à utiliser.

http://guides.library.duke.edu/vis_types [en anglais]

\section{D3 - Data-Driven Documents}

D3 est une bibliothèque graphique en langage JavaScript qui permet de générer de nombreuses typologies de visualisations de données. Le projet, porté par Mike Bostock, a succédé à un ancien prototype développé par le Groupe de visualisation de l'Université de Stanford et mise sur la compatibilité avec les standards du web. Le site propose aussi une galerie de visualisations qui offre une réelle source d'inspiration'. http://d3js.org/ [en anglais]

\section{NOTES}

1. «Story of the world» : http://www.bbc.co.uk/programmes/p00cgkfk 2010 ; « Teaching to see » : http://teachingtosee.org/. Site d'Edward Tufte : http://www.edwardtufte.com/

2. Présentation de Gaëtan Gaborit, chargé de mission pour l'Observatoire économique et social de la région Pays de Loire. 
3. Une présentation du travail de Jacques Bertin est disponible sur le site de l'École des hautes études en sciences sociales (EHESS) : http://lodel.ehess.fr/

4. «Graphical Perception and Graphical Methods for Analyzing Scientific Data» in Science, New Series, vol. 229, n4716 (1987), p. 828-833. Disponible sur le site de la School of Information de l'Université de Berkeley, en Californie : http://www.ischool.berkeley.edu/

5. Production individuelle de $\mathrm{M}^{\mathrm{me}}$ Teresa Elms : http://elms.wordpress.com

6. Production individuelle de Krisztina Szucs et Mate Cziner : http://www.szucskrisztina.hu

7. On peut aussi citer, en exemple, le simulateur de l'Indice composite d'apprentissage. Cet outil, proposé jusqu'en 2010 par le Conseil canadien de l'apprentissage, permettait aux différentes communes d'estimer l'impact de leurs politiques en matière d'apprentissage tout au long de la vie : voir Laughlin, J. et Lachance, M., «L'indice composite d'apprentissage du Canada. Au-delà d'un classement des villes “intelligentes" ", Revue internationale d'éducation de Sèvres, $\mathrm{n}^{\circ} 54$ (2010), p. 71-81. http://ries.revues.org/958/

8. Pour d'autres exemples de visualisation de données dans le data-journalisme, voir les lauréats de l'édition 2014 des Data Journalism Awards : http://www.globaleditorsnetwork.org/

9. Pour d'autres sources d'inspiration, se référer aux nombreuses plateformes de publication d'infographies comme http://www.visualizing.org/, http://visual.ly, http://infogr.am/, http:// www.informationisbeautiful.net/ etc.

\section{INDEX}

Mots-clés : visualisation de données, infographie, données statistiques

Keywords : data visualisation, computer graphics, statistical data

Palabras claves : visualización de datos, infografía, datos estadísticos

\section{AUTEUR}

\section{FEDERICA MINICHIELLO}

Federica Minichiello est chargée de veille au centre de ressources et d'ingénierie documentaires (CRID) du Centre international d'études pédagogiques (CIEP). 